\title{
EUV SOURCES AND TRANSIENTS DETECTED BY THE
}

\section{ALEXIS SATELLITE}

\author{
D. ROUSSEL-DUPRÉ ${ }^{1}$, T. PFAFMAN ${ }^{2}$, \\ J. BLOCH ${ }^{1}$ AND J. THEILER ${ }^{1}$ \\ ${ }^{1}$ Los Alamos National Laboratory \\ ${ }^{2}$ Stanford University
}

\section{Introduction}

Because ALEXIS is a spinning satellite, it is an ideal platform with which to study the time variability of the EUV cosmos. The main thrusts of this effort are to 1) detect EUV sources at known and unknown locations, 2) provide notification of transients in near real time to enable immediate follow-up from other observatories, and 3) create a time history of observed sources for comparison with previously published catalogs to aid in determining long duration variability from EUV sources.

\section{Real Time Point Source Determination}

Currently, the 12, 24 and 48 hour accumulation sky maps are searched as a routine part of the automated post-pass data processing. Eight on-line databases (Einstein Slew, EUVE, ROSAT-2RE, Yale Bright Star, Downes CV, TOAD CV, Gliese, and White Dwarf catalogs) are then automatically cross correlated for likely counterparts. Point source locations, source fit parameters and potential counterpart information are then sent via e-mail to the science team. Summary tables with this pertinent information and maps for these position are accessible at

http://nis-www.lanl.gov/nis-projects/alexis/

to the satellite team for evaluation. A pager alerts the on-duty scientist to significant transient detections found in the most recent data. The web page also contains a list of locations for 1) 40 sources with good potential for detection by ALEXIS spanning a range of system types (i.e., CV's, RS CVn's, ALGOL systems, flare stars, etc.), 2) 12 EUV bright sources and 
3) associated near-by blank fields as control locations, the count rate if it exceeds a certain threshold in a one degree box and other information about the detection are kept in a database. This can then be searched online to provide information about possible long term variability from these systems as well as use the blank field locations to provide a quick-and-dirty idea about the detection statistics. In addition to the on-line catalogs, we also use SIMBAD to search for likely candidates and the WEB SKYVIEW page to compare with other catalogs and other observations from various experiments. If a source passes all of the credibility tests, we then send out e-mail alerts to variable star observers around the world requesting ground based observations to help identify the optical counterpart.

\section{Types of Point Sources}

ALEXIS observes both steady sources (mostly bright white dwarfs) and transients. The transient or variable sources observed to date are 1) cataclysmic variables: VW Hyi, U Gem, AR UMa, 2) flare stars: Alpha Cen, RE J2353-702 and 3) unknown short duration transients. The cataclysmic variables show significant variability between outbursts both in overall brightness and spectral character. In addition, because of the continuous monitoring capability of ALEXIS, 2 EUV outbursts or "flashes" have been observed four days in advance of the optical turn-on for VW Hyi. Forty high probability unidentified short duration transients have been observed in an 11 month period. These unknown transients have durations ranging from 4-48 hours, and have no clear optical counterpart. Four of these systems have been observed by EUVE for as long as 24 hours without detection, although by the time EUVE was on target, the source was no longer bright in the ALEXIS instruments.

\section{Summary}

Because the ALEXIS attitude reconstruction algorithm has been evolving and changing through December 1995, the sky maps that are currently available and that have been searched for point sources have not been produced systematically with the same software. Therefore, it is difficult to ascertain the ALEXIS transient event rate, although the best estimate is about one per week. In the future, we will be reprocessing all archived data which will allow us to systematically determine the noise threshold level. Then the detailed source catalog including variability information can be generated and a better determination of the transient rate observed in each energy channel. 


\section{Acknowledgements}

These results represent the dedicated efforts of C. Little, M. Kennison, K. Ramsey, S. Ryan, S. Fletcher, B. Dunne, A. McNeil, P. Patel, P. Ratzlaff, S. Stem, and J. Wren. This work was supported by the Department of Energy. 